\begin{tabular}{|c|c|}
\hline Title & $\begin{array}{l}\text { Exosomes isolated from sera of mice fed Lactobacillus strains affect inflammatory cy tokine production in macrophages } \\
\text { in vitro }\end{array}$ \\
\hline Author(s) & A oki-Y oshida, A y ako; Saito, Shinichi; Tsuruta, Takeshi; Ohsumi, A risa; Tsunoda, Hinako; Sonoyama, Kei \\
\hline Citation & $\begin{array}{l}\text { Biochemical and biophysical research communications, 489(2), 248-254 } \\
\text { https://doi.org/10.1016j.bbrc.2017.05.152 }\end{array}$ \\
\hline Issue Date & 2017-07-22 \\
\hline Doc URL & http:/hdl.handle.net/2115/1028 \\
\hline Rights & $\begin{array}{l}\text { (C) } 2017 \text { Elsevier Inc. This manuscript version is made available under the CC-BY-NC-ND } 4.0 \text { license } \\
\text { http://creativecommons.org/icenses/fy-nc-nd/4.0/ }\end{array}$ \\
\hline Rights(URL) & http://reativecommons.org/icenses/by-nc-nd/4.0/ \\
\hline Type & article (author version) \\
\hline Additional Information & There are other files related to this item in HUSCAP. Check the above URL. \\
\hline File Information & A oki2017BBRC.pdf (article) \\
\hline
\end{tabular}

Instructions for use 


\section{Exosomes isolated from sera of mice fed Lactobacillus strains affect inflammatory cytokine production in macrophages in vitro}

Ayako Aoki-Yoshida ${ }^{\mathrm{a},{ }^{*}}$, Shinichi Saito ${ }^{\mathrm{b}, *}$, Takeshi Tsuruta $^{\mathrm{c}}$, Arisa Ohsumi ${ }^{\mathrm{d}}$, Hinako Tsunoda ${ }^{\mathrm{d}}$ and Kei Sonoyama ${ }^{\mathrm{e}, \dagger}$

${ }^{a}$ Graduate School of Agricultural and Life Sciences, The University of Tokyo, Tokyo 113-8657, Japan

${ }^{\mathrm{b}}$ Graduate School of Medicine, Yamaguchi University, Ube 755-8505, Japan

${ }^{\mathrm{c}}$ Graduate School of Environmental and Life Science, Okayama University, Okayama 700-8530, Japan

${ }^{\mathrm{d}}$ Graduate School of Life Sciences, Hokkaido University, Sapporo 060-8589, Japan

${ }^{\mathrm{e}}$ Research Faculty of Agriculture, Hokkaido University, Sapporo 060-8589, Japan

* These authors contributed equally to this study.

${ }^{\dagger}$ Corresponding author, Laboratory of Food Biochemistry, Research Faculty of Agriculture, Hokkaido University, Kita-9 Nishi-9, Kita-ku, Sapporo 060-8589, Japan

Tel \& Fax: +81-11-706-2496; E-mail: ksnym@chem.agr.hokudai.ac.jp (K. Sonoyama).

No. of words: 4,325

No. of Figures: 4

No. of Supplementary Table: 2 


\begin{abstract}
Orally administered Lactobacillus strains, including L. plantarum No.14 and L. rhamnosus GG, reportedly reduce inflammatory cytokine production in mice. The present study tested our idea that circulating exosomes mediate the action of Lactobacillus strains. The lipopolysaccharide-induced production of TNF- $\alpha$ and IL-6 in vitro was attenuated in peritoneal exudate cells (PECs) isolated from C57BL/6N mice that had been fed L. plantarum No.14. When PECs were cultured for $24 \mathrm{~h}$ with exosomes isolated from the serum of mice fed L. plantarum No.14 or L. rhamnosus GG, accumulation of both TNF- $\alpha$ and of the corresponding mRNA was lowered. Growth in the presence of these exosomes also decreased the production of TNF- $\alpha$ and IL- 6 by the murine macrophage cell line RAW264.7. In contrast, supplementation with exosome-depleted serum of mice fed L. plantarum No.14 or $L$. rhamnosus GG failed to affect the production of TNF- $\alpha$ and IL-6 by RAW264.7 cells. When PECs and RAW264.7 cells were cultured for $24 \mathrm{~h}$ with PKH67-labeled exosomes isolated from murine serum, fluorescent signal was observed inside the cells, suggesting that these cells incorporate serum exosomes. We propose that the anti-inflammatory activity of orally administered L. plantarum No.14 and L. rhamnosus GG is mediated, at least in part, by circulating exosomes.
\end{abstract}

Keywords: probiotics; inflammation; TNF- $\alpha$; IL-6; exosome 


\section{Introduction}

Probiotics are live microorganisms that, when administered in adequate amounts, confer a health benefit on the host [1]. To date, human and animal studies have reported that several orally administered bacterial strains, predominantly from the genera Lactobacillus and Bifidobacterium, exert beneficial effects on host health. However, the exact mechanism of action of these bacterial strains still remains unclear. We previously showed that oral administration of Lactobacillus plantarum No.14 reduces white adipocyte size in high-fat diet-induced obese mice [2]. Oral administration of this bacterial strain also has been reported to reduce body fat in healthy human volunteers [3]. In addition, we observed that feeding of $L$. plantarum No.14 to KK/Ta mice, an animal model of type 2 diabetes, decreases adiposity and improves insulin sensitivity [4]. Notably, the levels of mRNAs encoding TNF- $\alpha$, IL-6, and MCP-1 in mesenteric adipose tissue also are reduced in KK/Ta mice fed L.plantarum No.14 [4]. Thus, oral administration of L. plantarum No.14 may ameliorate adipose tissue inflammation, which might in turn improve insulin sensitivity. However, the mechanism by which L. plantarum No.14 affects adiposity and inflammation remains unknown.

Extracellular vesicles (EVs), which consist of a lipid bilayer membrane containing various biomolecules, are released by almost every cell type, and can be detected in the extracellular space and various biological fluids [5, 6]. EVs are known to transport their cargoes, including proteins, mRNAs, and microRNAs (miRNAs), from parental cells to recipient cells; EVs therefore are thought to play a role in regulating physiology and pathophysiology by mediating cell-cell communication [5, 6]. Indeed, EVs isolated from different biological fluids have been reported to modulate immune function [7-10]. These findings led us to speculate that the health-promoting actions of probiotics are mediated by circulating EVs. The present study tested our idea that the anti-inflammatory action of L. plantarum No.14 is mediated by circulating exosomes, a smaller type of EV that have diameters ranging from 30-100 $\mathrm{nm}[5,6]$. In addition, we previously demonstrated that adiponectin, an adipocyte-derived anti-inflammatory protein [11], is partially associated with exosomes in 
murine serum [12]. Therefore, we also tested whether adiponectin is involved in the action of circulating exosomes.

\section{Materials and methods}

\subsection{Bacterial strains and culture conditions}

L. plantarum No.14 was donated by Momoya Co., Ltd. L. plantarum type strain (JCM 1149T) and L. rhamnosus GG (ATCC 53103) were obtained from the Japan Collection of Microorganisms and the American Type Culture Collection, respectively. These bacteria were grown in De Man, Rogosa, and Sharpe broth (Difco Laboratories) at $37^{\circ} \mathrm{C}$ for $18 \mathrm{~h}$. Cultured bacteria were washed and resuspended in phosphate-buffered saline (PBS) and employed for oral administration in mice.

\subsection{Animal experiments}

All study protocols were approved by the Animal Use Committee of Hokkaido University (approval no. 14-0028). Animals were maintained in accordance with the Hokkaido University guidelines for the care and use of laboratory animals. Female C57BL/6N CrSlc mice were purchased from Japan SLC. Mice were housed in standard plastic cages in a temperature-controlled $\left(23 \pm 2{ }^{\circ} \mathrm{C}\right)$ room under a 12-h light/dark cycle and were allowed free access to water and standard chow diet (MF, Oriental Yeast). In experiment 1, after $7 \mathrm{~d}$ of consuming the diet, mice were divided into three groups (4 mice in each group). Each animal was then orally administered with either L. plantarum type strain $\left(10^{8}\right.$ colony-forming units $[\mathrm{cfu}] /$ mouse), L. plantarum No.14 (10 $\mathrm{cfu} /$ mouse), or vehicle (PBS) on a daily basis for $7 \mathrm{~d}$. On the last day of dosing, mice were euthanized by decapitation. Thereafter, peritoneal macrophages (peritoneal exudate cells, PECs) were obtained and counted as previously described [13] and then subjected to the cell culture experiments described below. In experiment 2 , after $7 \mathrm{~d}$ of consuming the diet, mice were divided into four groups (6 mice in each group). Each animal was then orally administered with either L. plantarum type strain (10 $\mathrm{cfu} / \mathrm{mouse})$, L. plantarum No.14 (10 $\left.{ }^{8} \mathrm{cfu} / \mathrm{mouse}\right)$, L. rhamnosus $\mathrm{GG}\left(10^{8} \mathrm{cfu} / \mathrm{mouse}\right)$, or 
vehicle (PBS) on a daily basis for $7 \mathrm{~d}$. On the last day of dosing, mice were anesthetized using intraperitoneal ketamine and xylazine ( 80 and $8 \mathrm{mg} / \mathrm{kg}$ body weight, respectively) and euthanized by bleeding aseptically by heart puncture. Following clotting, the serum supernatants were obtained by centrifugation and then subjected to exosome isolation as described below. PECs were obtained (as described above) from a separate group of treatment-naïve mice and then subjected to the cell culture experiments described below. In experiment 3, mice were fed the Lactobacillus strains, and exosomes were obtained from sera, as described in experiment 2, and then subjected to the cell culture experiments described below. In experiment 4, mice were fed the standard chow diet and then euthanized, and the serum exosomes were obtained as in experiment 2 and then subjected to PKH67 labeling as described below.

\subsection{Exosome isolation and PKH67 labeling}

Exosomes were isolated from serum samples using the ExoQuick Exosome Precipitation Solution (System Biosciences) according to the manufacturer's instructions. The precipitated exosomes were suspended in a volume of PBS equivalent to that of the original serum sample, and the suspension was then stored at $-80{ }^{\circ} \mathrm{C}$ until use. This suspension of exosomes was referred to as the $100 \%$ exosome fraction. The protein concentration in the exosome fraction was determined by the BCA protein assay (Thermo Scientific) according to the manufacturer's instructions. For fluorescent labeling, a volume of the exosome fraction equivalent to $100 \mu \mathrm{g}$ of protein was treated with the PKH67 Green Fluorescent Cell Linker Mini Kit (Sigma) according to the manufacturer's instructions, except that the labeled exosomes were separated from the labeling solution by ultrafiltration using a Vivaspin 6 (50-kDa cutoff; Sartorius). For use in control experiments, PBS was also treated in parallel with the exosome suspension.

\subsection{Cell culture}


PECs were seeded into each well of 96-well culture plates at $1.25 \times 10^{4}$ cells per well (experiment 1 ) or into 6 -well culture plates at $2.5 \times 10^{5}$ cells per well (experiment 2 ) using serum-free AIM V medium (Gibco) as the basal medium. RAW264.7 cells were obtained from the Japan Collection of Microorganisms and maintained in a basal medium composed of Dulbecco's modified Eagle's medium supplemented with 10\% fetal calf serum (FCS), 100 $\mathrm{U} / \mathrm{mL}$ penicillin, $100 \mu \mathrm{g} / \mathrm{mL}$ streptomycin, and $50 \mu \mathrm{g} / \mathrm{mL}$ gentamicin. The cells were cultured at $37{ }^{\circ} \mathrm{C}$ in a humidified atmosphere containing $5 \% \mathrm{CO}_{2}$.

\subsection{Experimental design}

In experiment 1, PECs were cultured in the basal medium supplemented with or without lipopolysaccharides from Salmonella abortus equi (LPS, Sigma) at a final concentration of 1 $\mu \mathrm{g} / \mathrm{mL}$ for $24 \mathrm{~h}$. In experiment 2, PECs were cultured as in experiment 1 except that the medium was further supplemented with a $10 \%$ exosome fraction isolated from mice fed $L$. plantarum type strain, L. plantarum No.14, L. rhamnosus GG, or vehicle, as described above. In experiment 3, RAW264.7 cells were seeded into each well of 48-well culture plates at $2.5 \times$ $10^{4}$ cells per well in the basal medium. After a 48-h incubation, cells were cultured in the medium supplemented with $0.5 \%$ exosome-depleted FCS for $24 \mathrm{~h}$. Thereafter, cells were cultured in the same medium supplemented with $10 \%$ exosome fraction isolated from mice fed L. plantarum type strain, L. plantarum No.14, L. rhamnosus GG, or vehicle, as described above, for $24 \mathrm{~h}$ under conditions where the medium was supplemented with or without LPS $(0.5 \mathrm{ng} / \mathrm{mL})$. Cells were also cultured in medium supplemented with $10 \%$ exosome-depleted serum prepared from mice fed L. plantarum type strain, L. plantarum No.14, L. rhamnosus GG, or vehicle. The exosome-depleted FCS was obtained by pelleting the exosomes via ultracentrifugation at $180,000 \times g$ for $18 \mathrm{~h}$ at $4{ }^{\circ} \mathrm{C}$. A portion of the murine serum samples was pooled in each group and then depleted for exosomes. Furthermore, portions of the exosome fraction and serum samples were subjected to ELISA for determination of adiponectin concentration as described below. In experiments 1-3, culture supernatants were subjected to ELISA for determination of TNF- $\alpha$ and IL- 6 concentrations as described below. 
In experiment 2, cells were subjected to RNA isolation followed by mRNA expression analysis as described below. In experiment 4, PECs and RAW264.7 cells were seeded into each well of 8-well chamber slides at a density of $1 \times 10^{5}$ cells/well and cultured overnight. PECs were cultured in the basal medium, while RAW264.7 cells were cultured in the basal medium supplemented with $10 \%$ exosome-depleted FCS. A volume of PKH67-labeled exosomes equivalent to $10 \mu \mathrm{g}$ of protein (or an equivalent volume of PKH67-treated PBS) was added to each well, and the cells were further cultured for $24 \mathrm{~h}$. After washing the wells with PBS, the cells were fixed with $4 \%$ formaldehyde and then observed under a fluorescent microscope (BX40, Olympus).

\subsection{ELISA}

The concentrations of TNF- $\alpha$ and IL-6 in the culture supernatant of PECs and RAW264.7 cells were determined using TNF alpha Mouse ELISA Kit (eBioscience) and IL-6 Mouse ELISA Kit (eBioscience), respectively, according to the manufacturers' instructions. The concentrations of adiponectin in the serum and exosome fraction were determined using Mouse Adiponectin/Acrp30 DuoSet ELISA (R\&D Systems) according to the manufacturer's instructions.

\subsection{Analysis of $m R N A$ expression}

Total RNA was isolated from PECs using a ReliaPrep RNA Cell Miniprep System (Promega) and reverse transcribed to generate first-strand cDNA using a ReverTra Ace qPCR RT kit (Toyobo) according to the manufacturers' instructions. To compare the steady-state levels of mRNAs encoding TNF- $\alpha$ and IL-6, real-time quantitative PCR (RT-qPCR) was performed using GeneAce SYBR qPCR Mix $\alpha$ No ROX (Nippon Gene) with a Thermal Cycler Dice TP800 (Takara Bio) according to the manufacturer's instructions. The sequences of primers used for RT-qPCR are shown in Supplementary Table 1. Relative mRNA expression levels for each sample were normalized to that of the transcript encoding $\beta$-actin. 


\subsection{Statistical analysis}

Results are presented as means and SEM. Mean values were compared among three or more groups by using a one-way analysis of variance (ANOVA) with a post-hoc Dunnett's multiple comparison test if significance was indicated. Data were analyzed using GraphPad Prism for Macintosh (version 6, GraphPad Software). $P$ values $<0.05$ were considered to indicate statistical significance.

\section{Results}

\subsection{Feeding L. plantarum No.14 decreases cytokine production in murine PECs (exp. 1)}

To test whether feeding Lactobacillus strains affects inflammatory cytokine production, PECs were isolated from mice and then incubated with or without LPS (Fig. 1A). The concentrations of TNF- $\alpha$ and IL-6 in the culture supernatants of PECs were significantly higher in the LPS-treated cells than in untreated cells (Fig. 1B and 1C). When the PECs were treated with LPS, TNF- $\alpha$ concentrations in the culture supernatants were significantly lower in the PECs from mice fed L. plantarum type strain or No.14 than in those from mice fed vehicle; TNF- $\alpha$ concentrations in the culture supernatants were also significantly lower in the PECs from mice fed L. plantarum No.14 than in those from mice fed L. plantarum type strain (Fig. 1B). The IL-6 concentrations were significantly lower in the PECs from mice fed $L$. plantarum No.14 than in those from mice fed vehicle or from mice fed L. plantarum type strain, whereas the concentrations were higher in the PECs from mice fed L. plantarum type strain than in those from mice fed vehicle (Fig. 1C).

\subsection{Exosomes isolated from sera of mice fed L. plantarum No.14 and L. rhamnosus $G G$} decrease cytokine production in murine PECs (exp. 2)

To test whether exosomes isolated from the sera of mice fed Lactobacillus strains affect inflammatory cytokine production, exosomes isolated from mice fed Lactobacillus strains were used to supplement the culture medium used for growing PECs isolated from other mice (Fig. 2A). The protein concentrations did not differ significantly in the $100 \%$ exosome 
fractions obtained from mice fed vehicle, L. plantarum type strain, L. plantarum No.14, or L. thamnosus GG $(2.71 \pm 0.42,3.06 \pm 0.73,2.85 \pm 0.61$, and $3.17 \pm 0.50 \mu \mathrm{g} / \mu \mathrm{L}$, respectively $)$. Upon LPS stimulation, the TNF- $\alpha$ concentrations in the culture supernatant and Tnf mRNA levels in the PECs were significantly lower in the PECs supplemented with exosomes from mice fed L. plantarum No.14 than in those from mice fed vehicle or L. plantarum type strain (Fig. 2B and 2C). Although the IL-6 concentrations in the culture supernatant of PECs supplemented with exosomes from mice fed L. plantarum No.14 did not differ significantly from those of mice fed vehicle or L. plantarum type strain (Fig. 2D), the Il6 mRNA levels were significantly lower in the PECs supplemented with exosomes from mice fed $L$. plantarum No.14 than in those from mice fed vehicle or L. plantarum type strain (Fig. 2E). The TNF- $\alpha$ concentrations in the culture supernatant were significantly higher in the PECs supplemented with exosomes from mice fed L. plantarum type strain than in those from mice fed vehicle, whereas there was no significant difference in the Tnf mRNA levels, IL-6 concentrations, or Il6 mRNA levels between the PECs supplemented with exosomes from mice fed vehicle and with exosomes from mice fed with L. plantarum type strain. The PECs supplemented with exosomes from mice fed L. rhamnosus GG showed significantly lower concentrations of TNF- $\alpha$ and IL-6 and of the respective mRNAs than PECs supplemented with exosomes from mice fed vehicle or L. plantarum type strain. No significant differences were found in any of these parameters between the PECs supplemented with exosomes from mice fed vehicle and PECs lacking exosome supplementation (data not shown).

\subsection{Exosomes isolated from sera of mice fed L. plantarum No.14 and L. rhamnosus GG decreases cytokine production in murine macrophage cell line RAW264.7 (exp. 3)}

To test whether exosomes isolated from the sera of mice fed Lactobacillus strains affect inflammatory cytokine production, the culture medium of murine macrophage cell line RAW264.7 was supplemented with exosomes (Fig. 3A). Upon LPS stimulation, both the TNF- $\alpha$ and IL-6 concentrations in the culture supernatant were significantly lower in the cells supplemented with exosomes from mice fed L. plantarum No.14 or L. rhamnosus GG than in 
those from mice fed vehicle or L. plantarum type strain (Fig. 3B and 3C). There was no significant difference in the TNF- $\alpha$ and IL- 6 concentrations between the cells supplemented with exosomes from mice fed vehicle and L. plantarum type strain. No significant difference was found in the TNF- $\alpha$ and IL- 6 concentrations between the cells supplemented with exosomes from mice fed vehicle and without exosome supplementation (data not shown). The TNF- $\alpha$ and IL- 6 concentrations did not significantly differ among the cells supplemented with exosome-depleted serum prepared from mice fed different Lactobacillus strains or vehicle (Fig. 3D and 3E). There was no significant difference in the adiponectin concentrations in the serum and exosome fractions between mice fed different Lactobacillus strains or vehicle (Supplementary Table 2).

3.4. Exosomes isolated from murine serum are incorporated into murine PECs and RAW264.7 cells (exp.4)

To test whether exosomes isolated from murine serum were incorporated into macrophages, the culture media of PECs and RAW264.7 cells were supplemented with PKH67-labeled exosomes (Fig. 4A). The PKH67 signal was observed in the PECs grown in medium supplemented with PKH67-labeled exosomes, whereas no signal was observed in cells grown in medium supplemented with PBS that had nominally been labeled with PKH67 (Fig. 4B). Likewise, the RAW264.7 cells grown in medium supplemented with PKH67-labeled exosomes showed a positive fluorescent signal, whereas the cells grown in medium supplemented with PKH67-treated PBS showed no signal (Fig. 4C).

\section{Discussion}

The present study showed that the oral administration of L. plantarum No.14 in C57BL/6N mice resulted in a decrease of LPS-induced production of TNF- $\alpha$ and IL-6 in PECs in vitro. These data were consistent with the results of our previous study, in which we demonstrated that oral administration of L. plantarum No.14 yielded decreased levels of mRNA encoding TNF- $\alpha$, IL-6 and MCP-1 in the mesenteric adipose tissue of obese KK/Ta mice [4]. Together, 
these results suggest that orally administered L. plantarum No.14 exerts anti-inflammatory activity in mice. To test whether this action is mediated by circulating exosomes, we isolated the exosomes from mouse serum and used the exosomal fraction to supplement the medium of PECs isolated from other mice. We found that the exosomes isolated from mice fed $L$. plantarum No.14 attenuated the LPS-induced accumulation of TNF- $\alpha$ and the TNF- $\alpha$-encoding transcript in the PECs; the effects on IL-6 expression were ambiguous. TNF- $\alpha$ and IL-6 production were also attenuated in the RAW264.7 cells grown in medium supplemented with exosomes isolated from mice fed L. plantarum No.14. These findings suggest that the anti-inflammatory action of orally administered L. plantarum No.14 is mediated, at least in part, by circulating exosomes. Given that exosome-depleted serum failed to alter the production of TNF- $\alpha$ and IL-6 in RAW264.7 cells, we infer that exosomes may play a major role in mediating the action of orally administered Lactobacillus strains.

Probiotic action is generally thought to be strain- but not species-dependent. Indeed, we found that oral administration of L. plantarum type strain, or cell growth in medium supplemented with exosomes isolated from mice fed the type strain, failed to reduce the production of TNF- $\alpha$ and IL-6 in PECs and RAW264.7 cells. In contrast, supplementation with exosomes isolated from mice fed L. rhamnosus GG, one of the best-known probiotic strains, reduced the production of TNF- $\alpha$ and IL-6 in PECs and RAW264.7 cells. Previous studies have demonstrated the anti-inflammatory action of orally administered L. rhamnosus GG. For instance, Ritze et al. showed that oral administration of L. rhamnosus GG for 8 weeks reduced hepatic mRNA levels of TNF- $\alpha$, IL-1 $\beta$, and IL-8R in mice fed a high-fructose diet [14]. Kim et al. observed that consumption of L.rhamnosus GG for 13 weeks reduced hepatic inflammation in mice fed a high-fat diet [15]. Zhang et al. reported that, although exopolysaccharides (EPS) isolated from L. rhamnosus GG failed to affect inflammatory cytokine production in murine 3T3-L1 preadipocytes in vitro, orally administered EPS reduced the expression of inflammatory cytokines in white adipose tissues and liver in diet-induced obese mice [16]; this result suggested indirect effects of EPS of L. rhamnosus 
GG on tissue inflammation. Considering the effect of exosomes isolated from mice fed $L$. rhamnosus GG on the production of inflammatory cytokines observed in the present study, it is conceivable that the circulating exosomes might contribute to the anti-inflammatory action of L.rhamnosus GG observed in the previous studies.

Adiponectin is a circulating protein with anti-inflammatory activity [11]. Given the previous demonstration that adiponectin is partially associated with exosomes in murine serum [12], we speculated that adiponectin is responsible for the anti-inflammatory activity of exosomes. However, we found no difference in adiponectin concentration, in either serum and exosome fractions, when comparing between mice fed Lactobacillus strains and those fed vehicle. Therefore, it seems unlikely that adiponectin contributes to the action of exosomes.

Circulating exosomes are the sum of the exosomes released from almost every cell type. Considering that intestinal epithelial cells are directly exposed to gut microbiota, the exosomes released from these epithelial cells may mediate the action of orally administered Lactobacillus strains. Indeed, intestinal epithelial cells have been reported to release exosomes that contribute to immune tolerance induction [7]. It also has been reported that gut microbiota influences the murine cecal expression signature of miRNAs [17], a type of exosome cargo. Thus, it seems likely that commensal bacteria, along with orally administered bacterial strains, influence the expression of exosome cargoes in intestinal epithelial cells, in turn altering the function of circulating exosomes. It will be of interest to determine whether exosomes released from intestinal epithelial cells cultured with microbial constituents and metabolites affect inflammatory cytokine production by macrophages.

The mechanism by which exosomes affect cytokine production in macrophages remains unclear. Chow et al. showed that breast cancer-derived exosomes are incorporated into macrophages and then activate NF-KB signaling in vitro and in vivo [18]. Those authors also found that proteins present on the surface of exosomes contribute to this activity [18]. Moon 
et al. demonstrated that caspase 3 encapsulated in exosomes derived from lung epithelial cells promotes cytokine production in macrophages via the ROCK1 pathway [19]. We found that exosomes isolated from murine serum are incorporated into PECs and RAW264.7 cells, suggesting that exosome cargoes such as surface and internal proteins and miRNAs may affect inflammatory signaling inside the recipient cells such as macrophages.

In conclusion, we demonstrated that exosomes isolated from the sera of mice fed $L$. plantarum No.14 or L. rhamnosus GG reduce inflammatory cytokine production in macrophages in vitro. We therefore propose that the anti-inflammatory activity of orally administered L. plantarum No.14 and L. rhamnosus GG is mediated, at least in part, by circulating exosomes.

\section{Acknowledgments}

This study was supported in part by the Regional Innovation Strategy Support Program of the MEXT; by the Center of Innovation Program from the MEXT and Japan Science and Technology Agency; and by the Akiyama Life Science Foundation. The authors have no conflict of interest to disclose. 


\section{References}

1. C. Hill, F. Guarner, G. Reid, et al., Expert consensus document. The International Scientific Association for Probiotics and Prebiotics consensus statement on the scope and appropriate use of the term probiotic. Nat. Rev. Gastroenterol. Hepatol. 11 (2014) 506-514.

2. N. Takemura, T. Okubo, K. Sonoyama, Lactobacillus plantarum strain No. 14 reduces adipocyte size in mice fed high-fat diet. Exp. Biol. Med. 235 (2010) 849-856.

3. Y. Nagata, Y. Kamimura, T. Saka, et al., Lactobacillus plantarum strain No. 14 suppresses human allergic reaction. J. Jpn. Soc. Food Sci. Technol. 55 (2008) 625-631 (in Japanese).

4. T. Okubo, N. Takemura, A. Yoshida, et al., KK/Ta mice mdministered Lactobacillus plantarum strain No. 14 have lower adiposity and higher insulin sensitivity. Biosci. Microbiota Food Health. 32 (2013) 93-100.

5. J.M. Pitt, G. Kroemer, L. Zitvogel, Extracellular vesicles: masters of intercellular communication and potential clinical interventions. J. Clin. Invest. 126 (2016) $1139-1143$.

6. M. Tkach, C. Théry, Communication by extracellular vesicles: Where we are and where we need to go. Cell 164 (2016) 1226-1232.

7. M. Karlsson, S. Lundin, U. Dahlgren, et al., "Tolerosomes” are produced by intestinal epithelial cells. Eur. J. Immunol. 31(2001) 2892-2900.

8. C. Admyre, J. Grunewald, J. Thyberg, et al., Exosomes with major histocompatibility complex class II and co-stimulatory molecules are present in human BAL fluid. Eur. Respir. J. 22 (2003) 578-583.

9. A. Sabapatha, C. Gercel-Taylor, D.D. Taylor, Specific isolation of placenta-derived exosomes from the circulation of pregnant women and their immunoregulatory consequences. Am. J. Reprod. Immunol. 56 (2006) 345-355.

10. Z.B. Deng, X. Zhuang, S. Ju, et al., Exosome-like nanoparticles from intestinal mucosal cells carry prostaglandin E2 and suppress activation of liver NKT cells. J. Immunol. 190 
(2013) 3579-3589.

11. N. Ouchi, K. Walsh, A novel role for adiponectin in the regulation of inflammation. Arterioscler. Throm. Vasc. 28 (2008) 1219-1221.

12. W. Phoonsawat, A. Aoki-Yoshida, T. Tsuruta, et al., Adiponectin is partially associated with exosomes in mouse serum. Biochem. Biophys. Res. Commun. 448 (2014)261-266.

13. S. Pirapatdit, E. Kishino, K. Fujita, et al., Dietary $\alpha$-linked galacto-oligosaccharide suppresses ovalbumin-induced allergic peritonitis in BALB/c mice. Biosci. Biotechnol. Biochem. 72 (2008) 1901-1907.

14. Y. Ritze, G. Bárdos, A. Claus, et al., Lactobacillus rhamnosus GG protects against non-alcoholic fatty liver disease in mice. PLoS One 9 (2014) e80169.

15. B. Kim, K.Y. Park, Y. Ji, et al., Protective effects of Lactobacillus rhamnosus GG against dyslipidemia in high-fat diet-induced obese mice. Biochem. Biophys. Res. Commun. 473 (2016) 530-536.

16. Z. Zhang, Z. Zhou, Y. Li, et al., Isolated exopolysaccharides from Lactobacillus rhamnosus GG alleviated adipogenesis mediated by TLR2 in mice. Sci. Rep. 6 (2016) 36083.

17. N. Singh, E.A. Shirdel, L. Waldron, et al., The murine caecal microRNA signature depends on the presence of the endogenous microbiota. Int. J. Biol. Sci. 8 (2012) 171-186.

18. A. Chow, W. Zhou, L. Liu, et al., Macrophage immunomodulation by breast cancer-derived exosomes requires Toll-like receptor 2-mediated activation of NF-кB. Sci. Rep. 4 (2014) 5750.

19. H.G. Moon, Y. Cao, J. Yang, et al., Lung epithelial cell-derived extracellular vesicles activate macrophage-mediated inflammatory responses via ROCK1 pathway. Cell Death Dis. 6 (2015) e2016. 
A
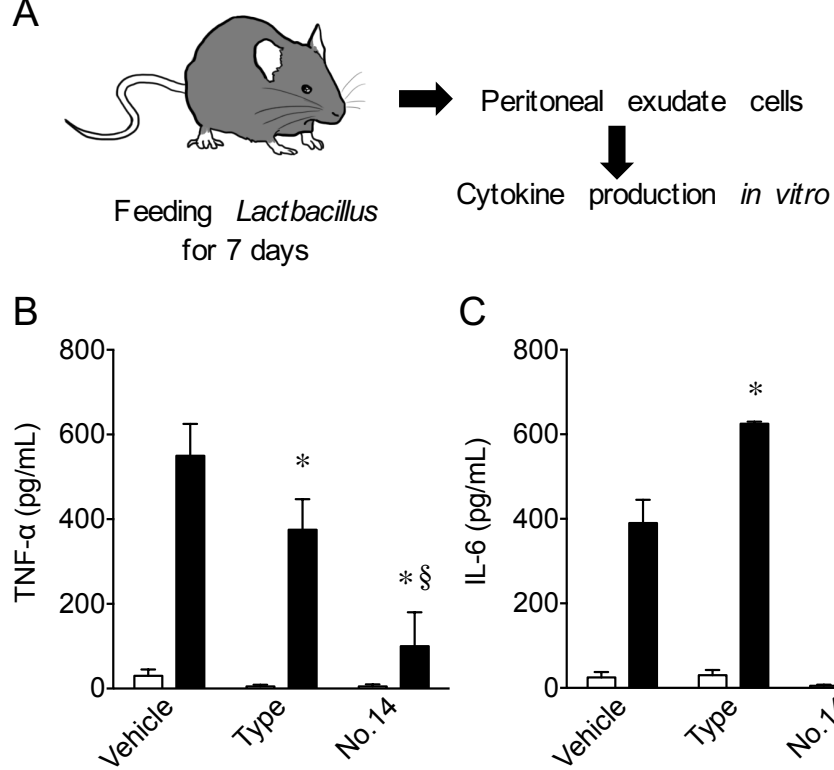

C

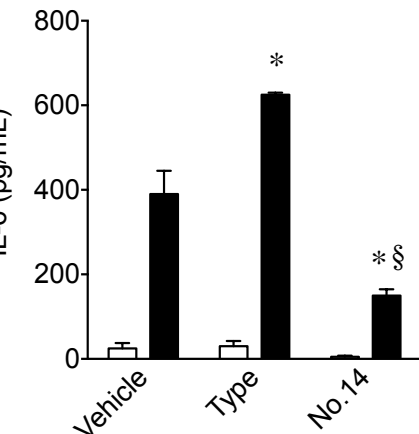

Figure 1. Feeding of mice with L. plantarum No.14 decreases cytokine production in murine PECs. $A$, Diagram of experiment $1 . B$ and $C$, TNF- $\alpha$ and IL-6 concentrations, respectively, in the culture supernatant of PECs treated with (closed column) or without (open column) LPS for $24 \mathrm{~h}$. Data are expressed as the mean and SEM of 4 mice. *, $p<0.05$ vs. vehicle; $\S, p<0.05$ vs. L. plantarum type strain, as estimated by one-way ANOVA followed by Dunnett's multiple comparison test.
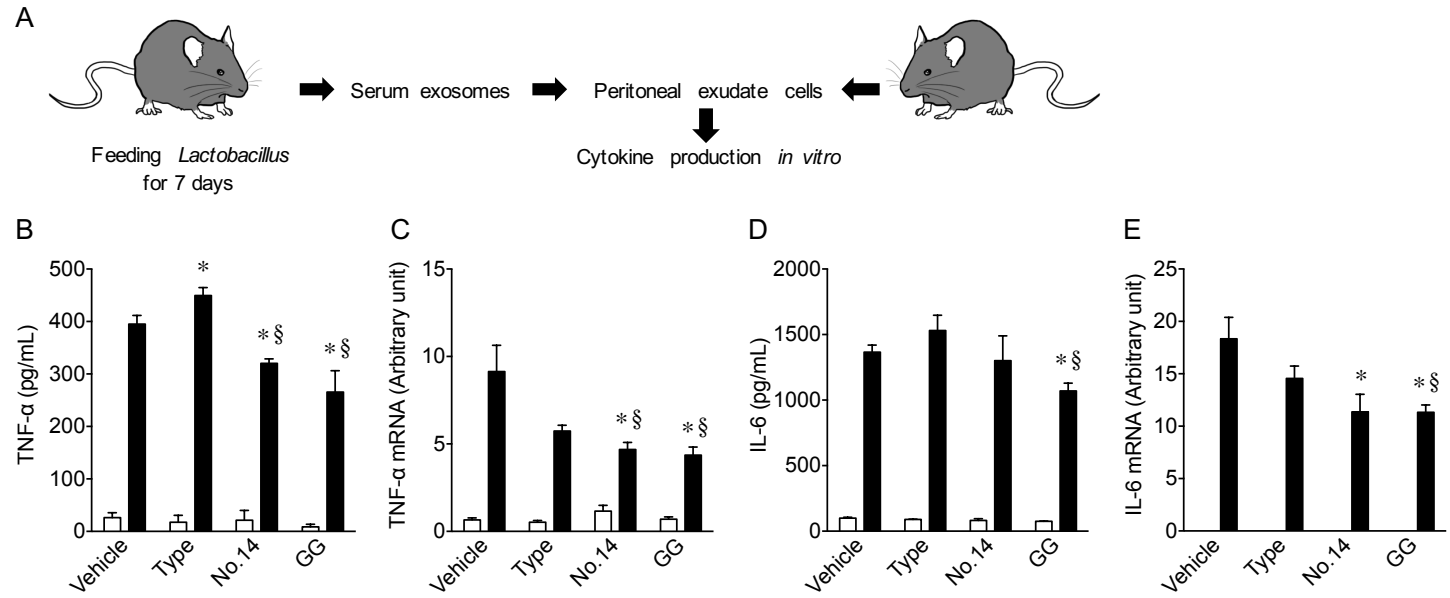

Figure 2. Exosomes isolated from the sera of mice fed L. plantarum No.14 or L. rhamnosus GG decrease cytokine production in murine PECs. A, Diagram of experiment 2. $B$ and $D$, TNF- $\alpha$ and IL-6 concentrations, respectively, in the culture supernatant of PECs treated with (closed column) or without (open column) LPS for $24 \mathrm{~h}$. $C$ and $E$, Relative levels of mRNAs encoding TNF- $\alpha$ and IL-6, respectively, in PECs treated with (closed column) or without (open column) LPS for $24 \mathrm{~h}$. Data are expressed as the mean and SEM of 6 mice. ${ }^{*}, p<0.05 v s$. vehicle; $\S, p<0.05 v s$. L. plantarum type strain, as estimated by one-way ANOVA followed by Dunnett's multiple comparison test. 

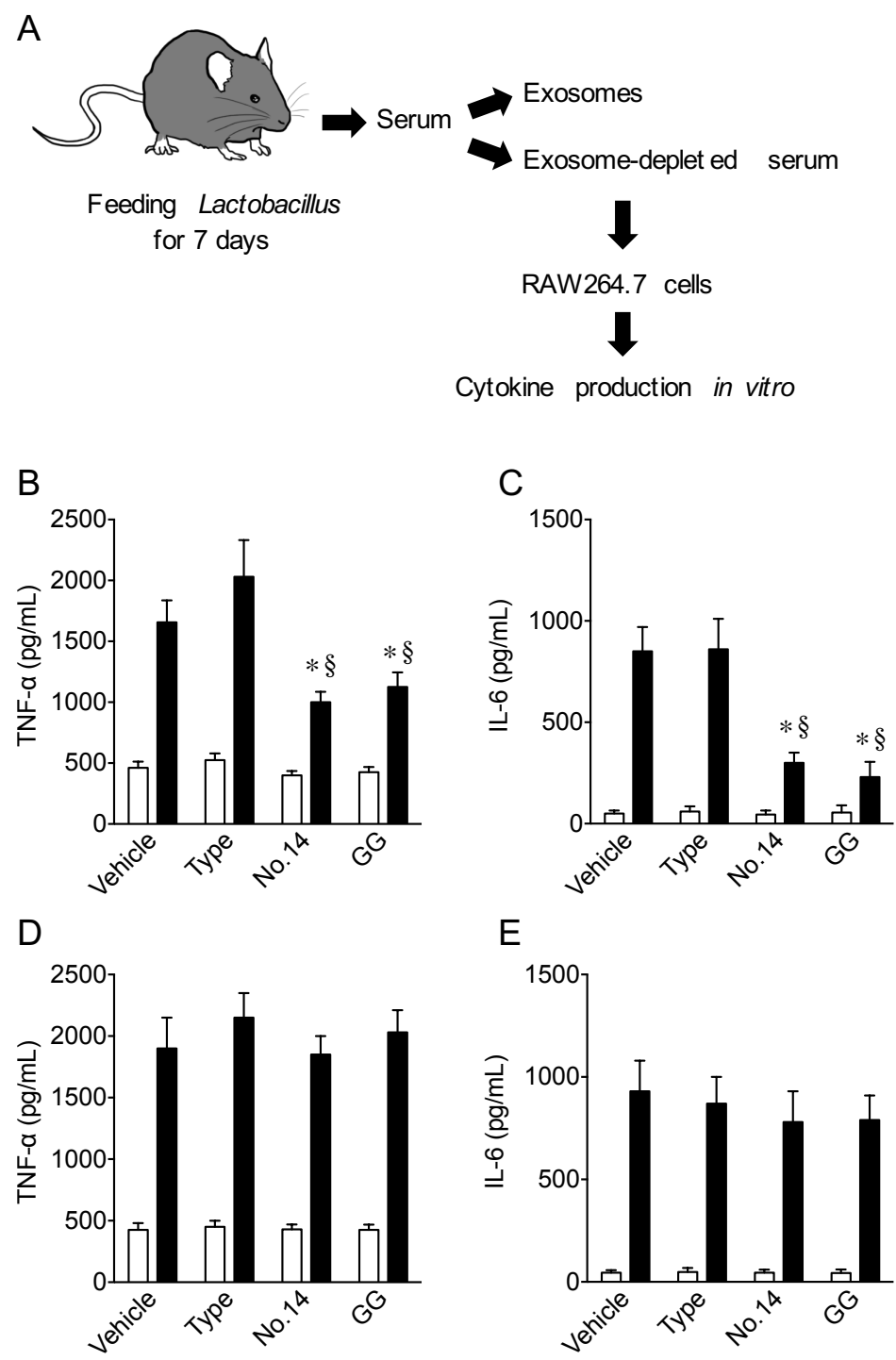

Figure 3. Exosomes isolated from the sera of mice fed L. plantarum No.14 or L. rhamnosus GG decrease cytokine production in murine macrophage cell line RAW264.7. A, Diagram of experiment 3. $B$ and $C$, Effect of supplementation with exosomes on TNF- $\alpha$ and IL-6 concentrations, respectively, in the culture supernatant of RAW264.7 cells treated with (closed column) or without (open column) LPS for $24 \mathrm{~h}$. Data are expressed as the mean and SEM of 6 mice. *,$p<0.05 v s$. vehicle; $\S, p<0.05 v s$. L. plantarum type strain, as estimated by one-way ANOVA followed by Dunnett's multiple comparison test. $D$ and $E$, Effect of supplementation with exosome-depleted serum on TNF- $\alpha$ and IL- 6 concentrations, respectively, in the culture supernatant of RAW264.7 cells treated with (closed column) or without (open column) LPS for $24 \mathrm{~h}$. Pooled serum samples from each group were subjected to depletion of exosomes and then added to the cells in triplicate; data are expressed as the mean and SEM of 3 wells. 

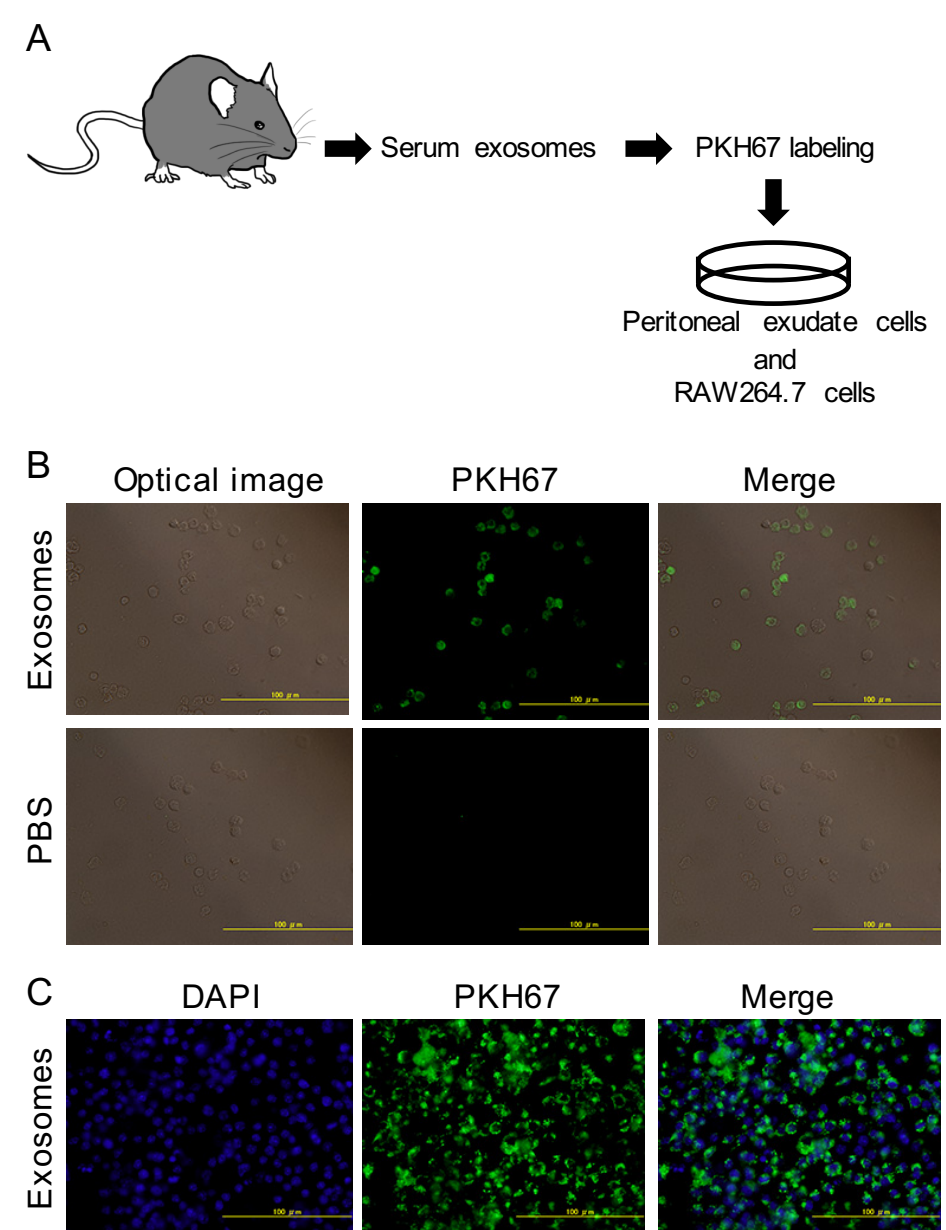

PKH67

Merge
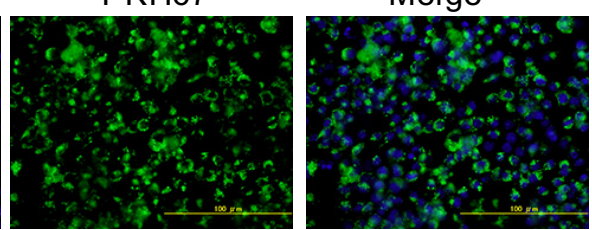

m
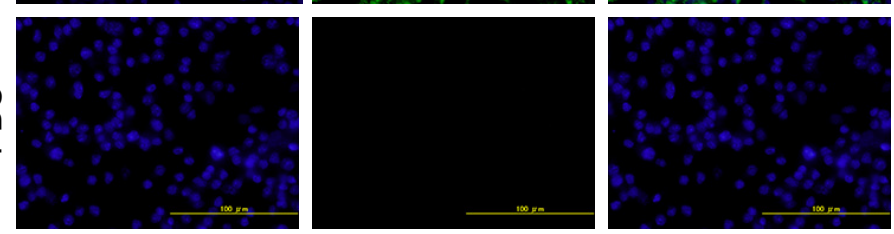

Figure 4. Exosomes isolated from murine serum are incorporated into murine PECs and RAW264.7 cells. $A$, Diagram of experiment 4. $B$ and $C$, Visualization of PKH67 signal in PECs and RAW264.7 cells, respectively. Bar, $100 \mu \mathrm{m}$. 
Supplementary Table 1 Primer sequences used for RT-qPCR analysis

\begin{tabular}{lll}
\hline Gene & Forward or Reverse & Sequence \\
\hline \multirow{2}{*}{ Tnf } & $\mathrm{F}$ & CATCTTCTCAAATTCGAGTGACAA \\
& $\mathrm{R}$ & TGGGAGTAGACAAGGTACAACCC \\
II6 & $\mathrm{F}$ & GAGGATACCACTCCCAACAGACC \\
& $\mathrm{R}$ & AAGTGCATCATCGTTGTTCATACA \\
\multirow{2}{*}{ Actb } & $\mathrm{F}$ & CTGGGACGATATGGAGAAGA \\
& $\mathrm{R}$ & AGAGGCATACAGGGACAACA \\
\hline
\end{tabular}

Supplementary Table 2 Adiponectin aconcentration in serum and exosome fraction of mice fed different Lactobacillus strains

\begin{tabular}{lcccc}
\hline & Veicle & L. plantarum type strain & L. plantarum No.14 & L. rhamnosus GG \\
\hline & \multicolumn{4}{c}{$\mu \mathrm{g} / \mathrm{mL}$} \\
Serum & $5.3 \pm 0.7$ & $5.1 \pm 0.2$ & $4.5 \pm 0.4$ & $4.5 \pm 0.6$ \\
Exosome & $0.054 \pm 0.010$ & $0.026 \pm 0.005$ & $0.046 \pm 0.010$ & $0.025 \pm 0.010$ \\
\hline
\end{tabular}

